\title{
Finite Element Modal Analysis of Four-link Combination Portal Crane
}

\author{
Yongfeng Zheng ${ }^{1}$, YouminHu${ }^{1}$, Bo $\mathrm{Wu}^{1 *}$, Pengxing $\mathrm{Yi}^{1}$, Jie $\mathrm{Liu}^{1}$ \\ ${ }^{1}$ State Key Laboratory of Digital Manufacturing Equipment \& Technology, Huazhong University of \\ Science and Technology, Wuhan, 430074, China \\ hnzyf08@163.com, (youmhwh, bowu)@hust.edu.cn
}

Keywords:Modal analysis, Four-link, Frequency, Crane.

Abstract: This paper take the four-link combination boom portal crane which typed MQ2533 of Weihua Group for study, According to the structural characteristics of boom system in the premise of reasonable and simplified model, it utilize the ANSYS software to build a four-bar linkage FEM model and does modal analysis, which evaluate the dynamic characteristics and provides a theoretical reference for the lightweight design of four-link mechanism.

\section{Introduction}

As a large lifting and transport equipment, portal crane is widely used in ports terminals 、 shipyard and construction of large hydropower stations, whose performance directly affects the efficiency of cargo transhipment. Portal crane can be divided into two kinds of four-link combination boom crane and single-jib portal crane in accordance with the form of arm posture ${ }^{[1]}$. The biggest advantage of the former is greater headroom below the boom under a certain lifting height, thus the total height of the four-link crane is lower than the latter, but the structure is complex, heavy weight. Currently, most of the domestic enterprise utilize the four-link combination jib cranes. Rotary segment equipped above the body base and the top rotating deployed a lifting boom and luffing mechanism. There is a running equipment, which runs beneath the bodies for the whole walking on the preset orbit. Four mechanisms operate synergistically, so as to complete equipment installation segment, cargo loading and unloading operations of the hull. Fig. 1 shows the portal crane of four-link combination boom.

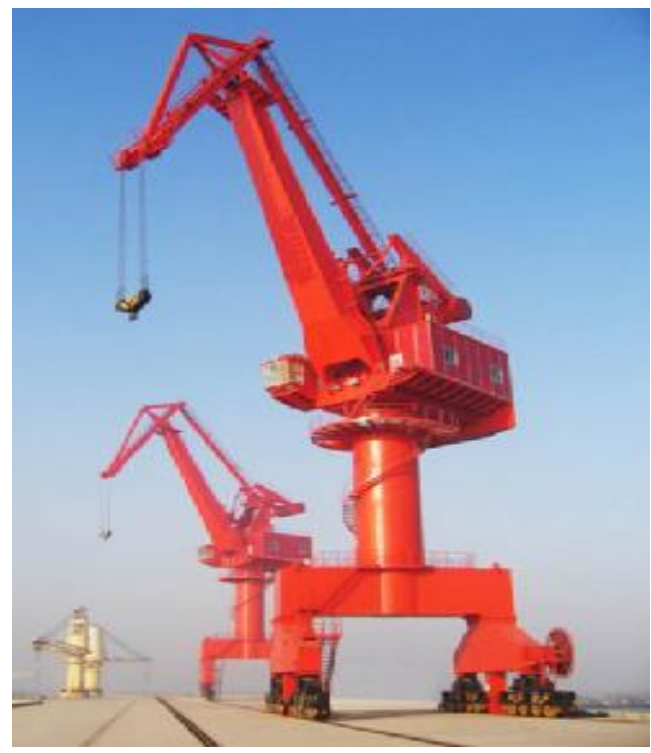

Fig.1 Portal crane of four-link combination boom

Mechanical structure need to meet the strength, stiffness and stability requirements, but also to 
meet the requirements of dynamic characteristics. Modal is the inherent vibration characteristics of mechanical structure, each mode has its corresponding natural frequencies and mode shapes. We can get the natural frequency and modes of the body between a band via obtaining each mode of four-bar linkage portal cranes, which can estimate the vibration response under certain incentives and avoid the excitation source frequency close to the natural frequency of relevant mechanism modes $^{[2]}$. Based on these, It is important to highlight thatthe methodology shown in this study can provide theoretical supports for early vibration failure and future structural optimization.

\section{FEM of four-link combination boom}

Meshing. MQ2533 portal crane is a box beams mainly welded by steel, the main technical parameters are shown in Tab.1, all welded parts are simplified as a continuous whole when building three-dimensional model of boom, and we ignore some fine structure of holes, triangular rib panels $^{[4]}$. In addition, we should attach the ladder railing systems, pipelines and other lubricants on the boom systemin the form of quality pointsuniformly.

Tab.1 Technical parameters of lifting system

\begin{tabular}{cc}
\hline Category & Parameters values \\
\hline Net height of the bboom boom system $(\mathrm{m})$ & 10 \\
Minimum amplitude $(\mathrm{m})$ & 9 \\
Maximum amplitude $(\mathrm{m})$ & 33 \\
Rated weight $(\mathrm{Kg})$ & 25000 \\
Rated lifting speed $(\mathrm{m} / \mathrm{s})$ & 0.67 \\
Lifting height $(\mathrm{m})$ & 42 \\
Working level & $\mathrm{M} 7$ \\
\hline
\end{tabular}

MQ2533 portal crane using Q345 which has a better strength. The elastic modulus of material is set for $2.06 \mathrm{e} 11 \mathrm{~Pa}$, Poisson is 0.28 , the material density is set for $7910 \mathrm{Kg} / \mathrm{m}^{3}$ according to the same principle of total mass ${ }^{[5]}$. This paper adoptsthe two-node box beam elements and line grid, dividing the model into 283 units and 293 nodes. FEM of the boom system is shown in Fig.2, and Fig.3 furthur explains the simplified model of four-link boom.

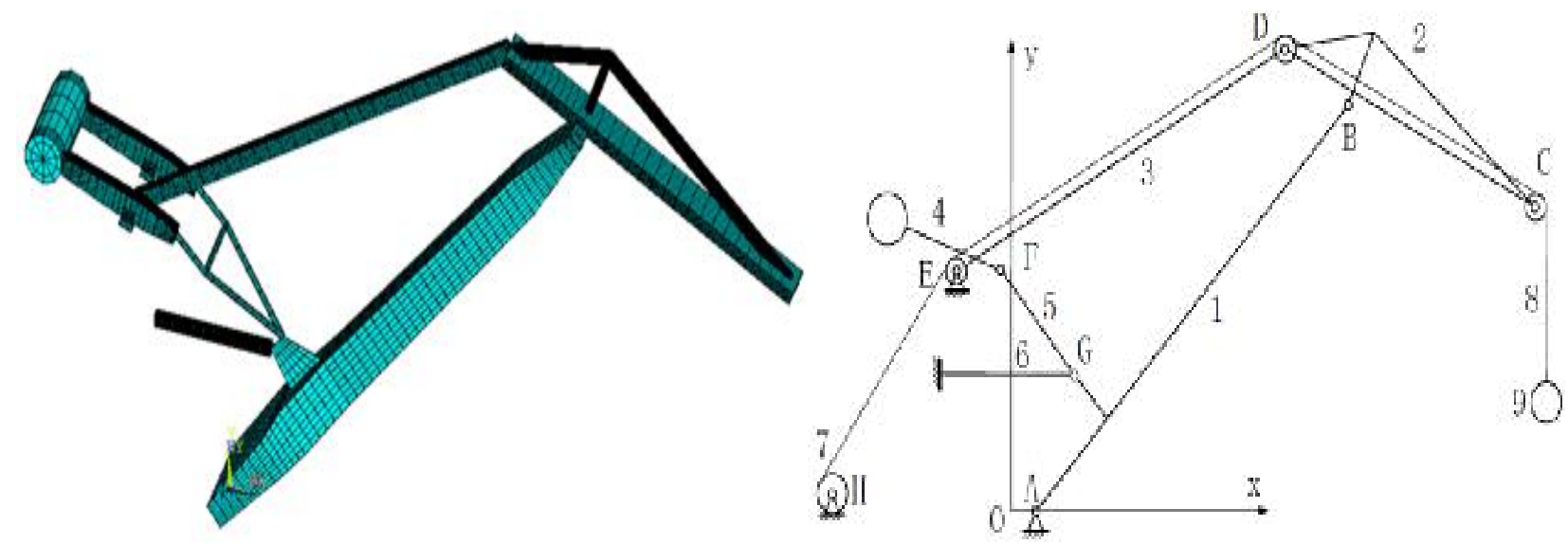

Fig.2 FEM of the boom systemFig.3 Simplified model of four-link boom

1-boom 2-cantilever 3-big drawbar 4-counterweight 5-small drawbar6-luffing gear-rack 7-drum 8-wire rope 9-loadA,B,C,D,E,F,G,H-the hinge point

Constraints imposed. This paper constraint UX, UY, UZ, ROTX, ROTY degree of freedom of some parts, which contain the lower hinge point of boom and big rodas well asthe hinge pointamong 
each link, then they can rotate only around the $\mathrm{Z}$ axis.At the same time, we should constraintall degrees of freedom of the left rack.

Modal analysis. This paper select the the type ofModal in ANSYS, then run the "Solve | Current LS"command, the corresponding frequency of each mode are shown in Tab.2. According to the structuraland operating characteristics of the portal crane,its execution units perform at a low speed running range, so the lower modes play a major role in affectingdynamic characteristics ${ }^{[6]}$. On the contrary, high-frequency modes of the structure has little effect and can be ignored. Here we only select the first four modal.

Tab.2The first 4-order natural frequency of the boom system

\begin{tabular}{ccccc}
\hline Order & 1 & 2 & 3 & 4 \\
\hline requency & 1.3703 & 1.8357 & 2.3344 & 3.0274 \\
\hline
\end{tabular}

Seen from the above table, we acknowledge that the first 4-order natural frequencies all exceed $1 \mathrm{~Hz}$, which demonstrate the four-link combination boom can meet the crane design criterion. Then, the first 4-order mode shapes of boom system are shown in Fig.4 to 7.

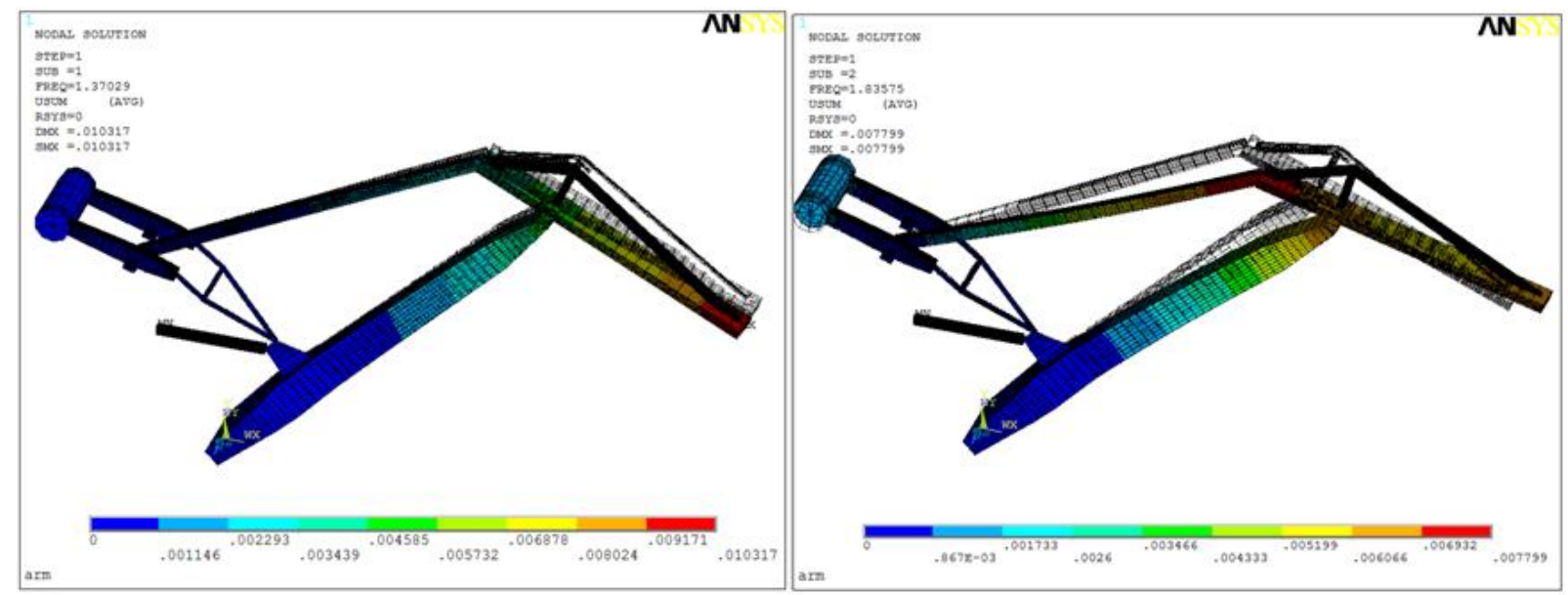

Fig.4 The first modal shapeFig.5 The second modal shape

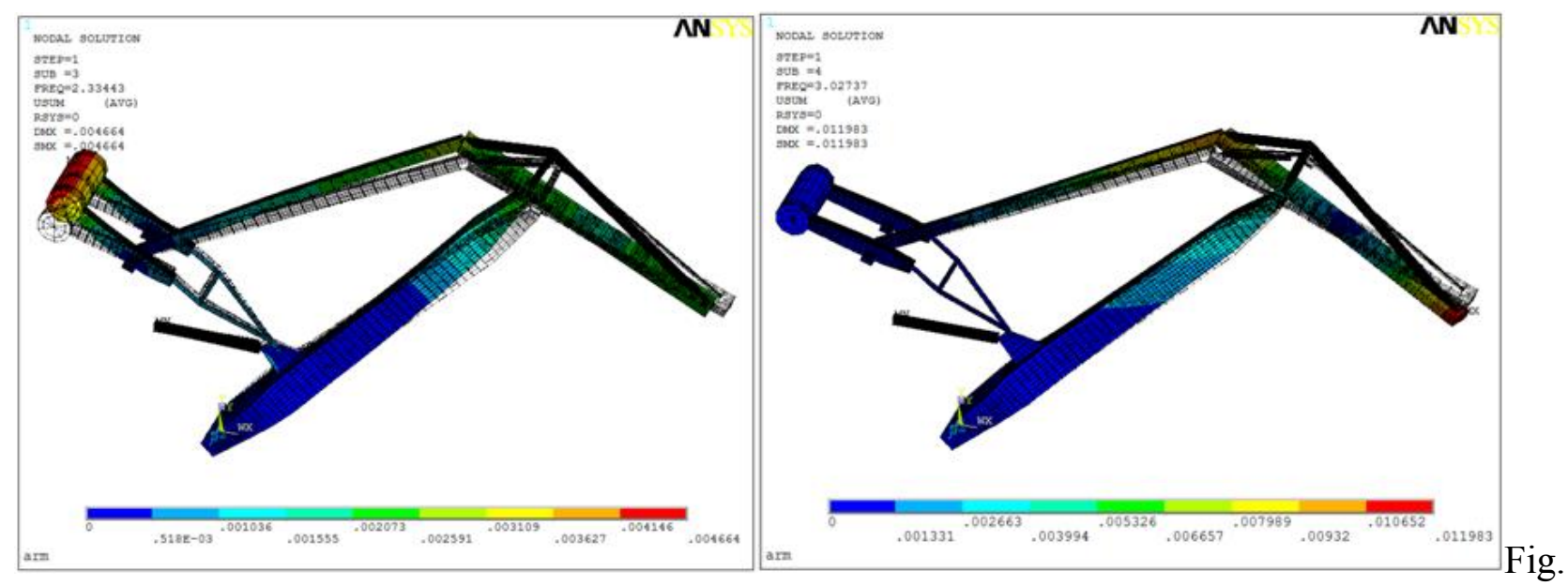

6 The third modal shapeFig.7 The fourth modal shape

Themain excitation of boom swing is load vibration and wind load in practical working process, and Fig.4 depicts the first modal shape, which shows the boom mechanism swing in the horizontal direction, the first-order natural frequency is $1.3703 \mathrm{~Hz}$. load vibration is mainly relative to the 
length of suspension rope and rotation speed, because this paper does not study the rotation case and ignores the wind load, we have no need to analysis the first modal shape.

Seen from Fig.5, the second-order modes is the boom system swing around the hinge point of small rod and Boom, the natural frequency is $1.8357 \mathrm{~Hz}$. It mainly caused by the lifting process in practice. So the operator shouldnot start in a high-speed or leapfrog to avoid large impact occurs.

Fig. 6 shows the third-order mode for swinging around the bottom hinge point of boom, the natural frequency is $2.3344 \mathrm{~Hz}$.It mainly caused by sling load swing during the amplitude variation. So the operator should try to avoid the valueswhich prone toresonanceduring the parameter setting,such asamplitude speed or sling loadheight which need to luff frequently.

Fig. 7 presents the fourth mode, which mainly shows the boom twisting, the natural frequency is $3.0274 \mathrm{~Hz}$. This mode shape is usually carried out in multiple conditions, such as rotating and lifting simultaneously in practical.

\section{Summary}

The modal results of four-linkcombination system of the portal crane provide a reference for dynamic analysis, stability analysis and lightweight design of the similarstructure. The first four natural frequencies of four-bar linkageof portal crane are more than $1 \mathrm{~Hz}$, indicating the boom system can meet the dynamic design criteria.In the course of the actual working, the operator shouldnot start in a high-speed or leapfrog to avoid large impact occurs. Furturemore,the operator should try to avoid the values which prone to resonance during the parameter setting.

\section{Acknowledgements}

I am very grateful toNational Key Technology Research and Development Program of the Ministry of Science and Technology, China, which has funded our research. The fund number is 2015BAF06B06 and 2015BAF01B04.

\section{References}

[1]Chen Xinquan, He Yanping, Li Hongliang, Tan Jia-hua1. Study on the dynamic load of rope and its effect on floating and stability of revolving crane vessel [J]. Journal of Shanghai Jiaotong University, 2010,44(6): 778-781.

[2] Yongfeng Zheng, Shicheng $\mathrm{Hu}$, Xiangjun Wang, Yongwang He. Finite element analysis and structural optimization of the reachstacker frame[J]. Journal of Mechanic Manufacturing, 2013,51(587): 18-20.

[3] Hector A. Tinoco, Diego A. Ocampo, Juan R. Sanz-Uribe.etc. Finite element modal analysis of the fruit-peduncle of Coffea arabica[J]. Computers and Electronics in Agriculture 108(2014)17-27.

[4] Liming Li. Practical guide of finite element analysis[M]. Tsinghua University Press, Beijing, 2005.1, 266-270.

[5]Xu Lixin, Yang Yuhu, Chang Zongyu. Modal Analysis on Transverse Vibration of Axially Moving RoHer Chain Coupled with Lumped Mass[J]. Journal of Central South University of Technology(English Edition), 2011,18(1)109-115.

[6] Shicheng Hu, Shuo Ouyang, Ningbo Zou, Xiangjun Wang. The electromechanical coupling dynamics modeling and analysis of portal crane's hoisting system[J]. Advanced Materials Research, v 952, 2014, 210-15. 